\title{
IR and Raman Investigation of Some Poly(acrylic) Acid Gels in Aqueous and Neutralized State
}

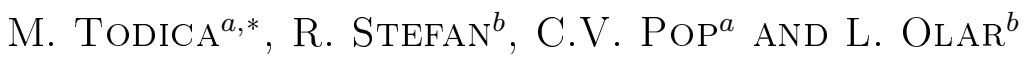 \\ a"Babes-Bolyai" University, Faculty of Physics, No. 1, M. Kogălniceanu Str., Cluj-Napoca, 400084, Romania \\ ${ }^{b}$ USAMV, Cluj Napoca, Romania \\ (Received October 3, 2014; in final form February 19, 2015)
}

\begin{abstract}
The modification of local polymeric conformation of poly(acrylic) acid (PAA), induced by hydration and neutralization with triethanol amine (TEA), was investigated by IR and Raman spectroscopy. The effect of water is the perturbation of hydrogen bonds established between different polymeric chains and the modification of the local conformation of the polymer. The neutralization with TEA affects the carboxylic groups and modifies the local conformation of the polymer. These modifications are correlated with the shift and the modification of the characteristics of IR and Raman spectra.
\end{abstract}

DOI: $10.12693 /$ APhysPolA.128.128

PACS: 82.70.Dd, 82.70.Gg, 82.70.Kj

\section{Introduction}

The use of the polymers in pharmaceutical industry as support for the medical drugs is actually an extended practice [1-3]. Often the polymers are used as thin membranes for the encapsulation of the active medical substances. Usually these products are designed for oral administration. The role of the polymeric capsule is to transport the drug in the desired region of the digestive tract, stomach, intestines or colon, and to ensure a progressive diffusion of the substance through the walls of the capsule. Finally the polymer is dissolved by the gastric liquids and then eliminated by physiological ways. Other use refers to the skin disease and in this case the product is presented under the gel form. The polymer represents the lattice which hosts the medical drug. Its role is to ensure the contact with the skin and progressive delivery of the active substance. Whatever the mode of use of the polymers is, these materials must be biocompatible with the tissues, must be removed by simple way from the tissues, or eliminated naturally by the living organisms, and must have no toxicity.

One of the polymers with large potential of applications in medicine, as mucoadhesive polymer or gels for the skin care, is the PAA [4-6]. This is a hydrophilic polymer from which gels can be obtained by simple hydration. The gels are structures with large amount of free spaces dispersed in the whole volume of the sample, where the medical active substance can be host. The product can be displayed directly on the skin, ensuring a good contact between the product and the tissues and a progressive delivery of the substance. The PAA gels have low consistency and viscosity, are enough adherent to the skin and can be removed by simple washing with water. Due to the low mechanical properties, some times it can be removed

\footnotetext{
* corresponding author; e-mail mihai.todica@phys.ubbcluj.ro
}

accidentally from the skin. This undesired situation can be avoided by increasing the mechanical properties, especially the viscosity.

The viscosity can be increased by cross linking the polymer, or by neutralization with a simple base, for instance the TEA [7]. The use of the second method offers the advantage of neutralization of residual acid groups of PAA remaining in the sample after the formation of the gel, but presents also the disadvantage of the introduction of a new undesired product in the sample, the base that is not well accepted by the tissues. From this point of view the neutralization must be done with high care, monitoring continuously the $\mathrm{pH}$ of the sample until a neutral product is obtained. Both the processes, the hydration and neutralization, can modify the conformation of the polymer. These modifications depend on the polymer and neutralizer concentration. During the hydration, the water molecules interact with the carbonyl groups of the polymer, and modify the hydrogen bonds between the chains. The local dynamics of the polymer increases and its state of aggregation changes dramatically compared with the initial state before hydration.

In the neutralization process the carboxylic groups are involved in chemical reactions with the base molecules, having as consequence the modification of the local conformation of the chains. The mechanical properties of the polymeric matrix, the delivery rate of the active substance are highly dependent on the structure of the host. Clarification of local modifications induced by hydration and neutralization of polymeric matrix represent important stages on the synthesis of such polymeric supports. The aim of our study is the observation of these modifications by IR and Raman spectroscopy.

\section{Experimental}

In dried state the PAA is a white powder with great affinity towards the water. The PAA gels were prepared by mixing for $4 \mathrm{~h}$ the polymer (molecular mass $104400 \mathrm{~g} / \mathrm{mol}$ ), in powder state with distilled water, 
at room temperature, until a homogeneous distribution of the polymer is obtained. The weight concentrations of the polymer in the solutions were $0.5 \%, 1 \%$, and $1.5 \% \mathrm{~g} / \mathrm{g}$. At higher concentration of polymer homogeneous gels cannot be obtained [8]. The neutralization was done with TEA in the proportion $1.5 / 1 \mathrm{~g} / \mathrm{g}$ TEA/PAA. At this ratio base/polymer the $\mathrm{pH}$ of the gel is about $6.5-7$. The FTIR absorption spectra were recorded in $400-4000 \mathrm{~cm}^{-1}$ spectral range with JASCO FTIR 4100 spectrometer. The Raman spectra were recorded with Equinox 55 spectrometer in the spectral range of $400-1800 \mathrm{~cm}^{-1}$. Excitation was done with $80 \mathrm{~mW}$ at $1024 \mathrm{~nm}$ laser source. A digital data acquisition is performed by the $\mathrm{PC}$ computer of the system.

\section{Results and discussions}

In dried state, before hydration, the PAA adopts a compact structure containing tightly coiled chains, with low dynamics, and low connectivity between them (Fig. 1a). We cannot speak about a real extended polymeric network. Many local conformations are possible, each of them being characterized by specific vibrations of the molecular bonds $[9,10]$. The vibration of the monomer, especially the vibration involving the carboxyl groups $(-\mathrm{COOH})$, are influenced by the neighbors of the monomer. In dried state, before the addition of water, each monomer has in its vicinity only polymeric monomers. The IR and Raman spectra are characterized by bands appearing at specific wave numbers, [11]. Every modification of the neighborhood of molecules is followed by the modification of the vibration of the molecular bonds. Such situations appear after hydration of PAA, in the gel state, or after neutralization.

The PAA gels can be obtained simply by dissolving the powder polymer in distilled water. The affinity towards the water is determined by the particular structure of the monomer, especially the presence of the bending group $(-\mathrm{OH})$ (Fig. 1c). This group, having an electric dipolar character, are the most susceptible to interact with the water molecule (which have also electric dipolar character). Due to this interaction the polymeric chains begin to uncoil partially, and become more freely to move. In this situation two events are more probable, the diffusion of the chains along the reptation tube with the apparition of temporary entanglements between two or more polymeric chains, and the apparition of hydrogen bonds between the bending oxygen atom of the $(-\mathrm{COOH})$ group of one chain and the $(-\mathrm{OH})$ group of the $(-\mathrm{COOH})$ of a neighboring chain [12]. The result is the apparition of a polymeric network with large free spaces where the water molecules can be trapped. This situation with two separate phases, the solvent and the polymer, corresponds to the gel phase [13]. The vicinity of the majority of the monomers, especially the vicinity of the bending groups $(-\mathrm{COOH})$ is different in the gel compared with the dried phase, and consequently, some vibrations are affected. The result is the modification of the IR and Raman spectra. In the gel state the entanglements between the polymeric chains have temporary character,
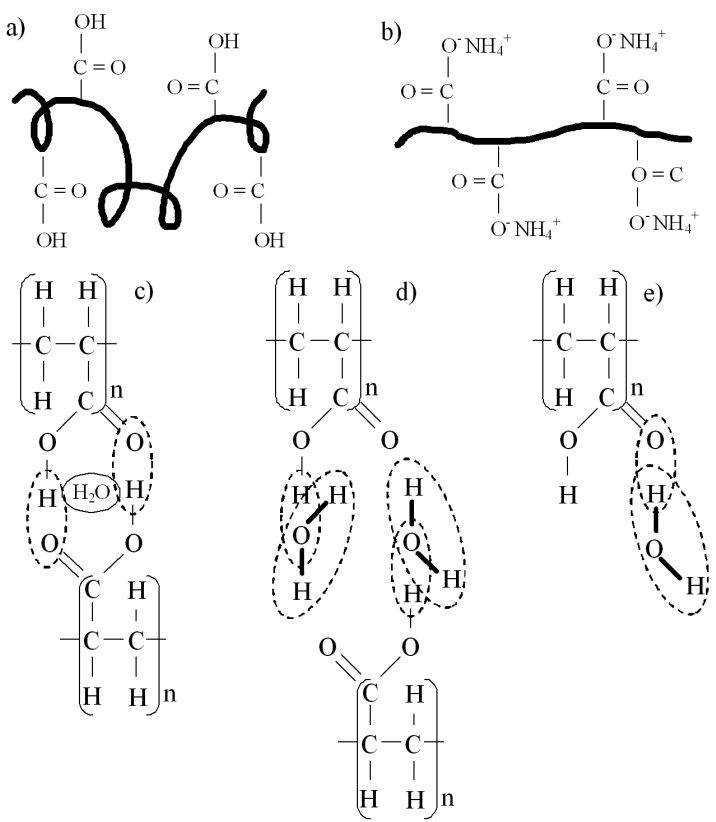

Fig. 1. Different conformations of PAA. (a) Coiled conformations of solid PAA; (b) uncoiled conformation of polymeric chains in the neutralized PAA gels; (c) possible inclusion of water molecules into PAA gel; free volume inclusion; (d) cyclic dimer; (e) hydrogen bond with (CO) groups not engaged in hydrogen bonds.

determined by the relatively high dynamics of the chains. For this reason the gels are characterized by low viscosity and weak mechanical properties. The temperature and the solvent concentration affect strongly this process. The network of the polymer becomes more stable, and the viscosity increases after neutralization with a given base, i.e. the TEA. In this chemical reaction the carboxylic group of PAA interacts with the TEA molecules, and the chains adopt an uncoiled conformation (Fig. 1b). The attachment of the amino group to the monomer reduces substantially the mobility of the chains, which explains the increase of the viscosity after neutralization. On the other hand, the vibrations of the carboxyl group involved in this reaction are affected, due to the amino group. We expect to observe some modifications of IR and Raman spectra after neutralization. As general observation until there, the hydration and neutralization of the PAA induces modifications on the vibrations of the monomer, especially of the carboxylic group. These vibrations are characteristics for each state of aggregation, solid, gel and neutralized gel, fact that justifies the comparative analysis of IR and Raman spectra of each kind of samples.

\subsection{Analysis of pure components}

\subsubsection{IR analysis of pure PAA}

For better understanding of the properties of the aqueous and neutralized gels we analysisd first the pure components, the PAA and the TEA, before hydration and neutralization. The IR spectra of PAA contain large 
bands, determined by the multitude of possible local conformation of the polymeric chains. Apart these large bands, in the IR spectrum of pure PAA in dried state, before hydration, we can observe an important sharp band at $1714 \mathrm{~cm}^{-1}$ (Fig. 2). This band is assigned to the vibrations of the carbonyl group of the carboxylic group of the monomer. Kim et al. observed this band at $1709 \mathrm{~cm}^{-1}$ and correlated it with the intermolecular hydrogen bonds among the carbonyl groups of the polymer [14]. As shown by other authors this band is the most affected by the conformational changes of the polymeric chains induced by hydration or neutralization $[12,14]$. Other bands appear at 629, 801, 1172, 1241, 1446, $1714 \mathrm{~cm}^{-1}$ (Fig. 2). The band at $629 \mathrm{~cm}^{-1}$ is assigned to $\delta(\mathrm{C}=\mathrm{O}$, trans $)$ [15]. The bands between 1130 and $1300 \mathrm{~cm}^{-1}$ are assigned to (-CO) stretching and $(-\mathrm{OH})$ bending vibrations $[12,16]$. The band $1446 \mathrm{~cm}^{-1}$ is assigned to $\delta\left(\mathrm{CH}_{2}\right),[12,15$, $17,18]$. We note there that the bands at $629 \mathrm{~cm}^{-1}$, $1172 \mathrm{~cm}^{-1}$ and $1446 \mathrm{~cm}^{-1}$ are IR and Raman active [15]. Ours data are in agreement with other works reported in literature $[12,15]$.

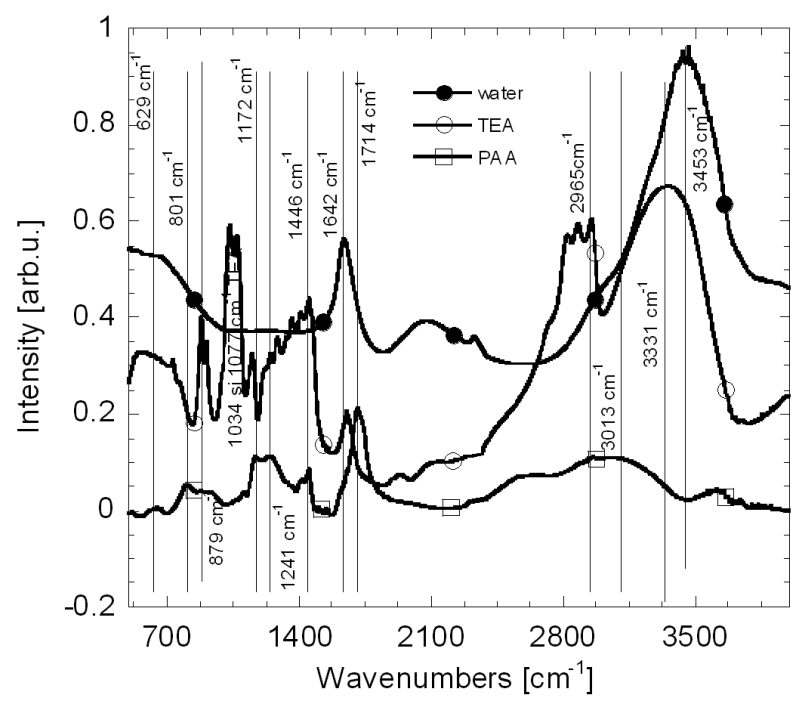

Fig. 2. IR spectra of PAA, TEA and water in pure state.

\subsubsection{IR analysis of pure TEA}

The IR spectrum of the pure TEA shows two narrow bands very close each to other at 879 respectively $905 \mathrm{~cm}^{-1}$, other very closed bands at 1034 and $1077 \mathrm{~cm}^{-1}$, single narrow band at $1146 \mathrm{~cm}^{-1}$, many bands superposed into a large one between 1275 and $1446 \mathrm{~cm}^{-1}$. The band at $1446 \mathrm{~cm}^{-1}$ can be seen also in the spectrum of pure PAA. We can observe also a narrow band at $1642 \mathrm{~cm}^{-1}$, a superposition between 2810 and $2965 \mathrm{~cm}^{-1}$ and a large band centered at $3331 \mathrm{~cm}^{-1}$ (Fig. 2). Detailed assignment of the bands can be established on the basis of experimental and theoretical results obtained by Koruda et al. on systems close to ours [19]. For instance the bands between 756 and $905 \mathrm{~cm}^{-1}$ are assigned to different skeletal vibration of $\left(\mathrm{C}_{3} \mathrm{NO}\right)$ group. The bands around $1000 \mathrm{~cm}^{-1}$ are assigned to vibrations of $(-\mathrm{OH})$ groups. The band at $1146 \mathrm{~cm}^{-1}$ is caused mainly by the $(\mathrm{NCH})$ bending. The band at $1240 \mathrm{~cm}^{-1}$ is assigned to $\nu$ vibrations of $(\mathrm{NCH})$ and $(\mathrm{HCH})$ bending motions. The band at $1398 \mathrm{~cm}^{-1}$ is assigned to $\nu$ symmetric $\mathrm{CH}$ vibration. The bands at $1446 \mathrm{~cm}^{-1}$ are mainly due to $(\mathrm{NCH})$ and $(\mathrm{HCH})$ bending motions which are coupled in-phase. The bands at $2965 \mathrm{~cm}^{-1}$ arise from pure $(\mathrm{C}-\mathrm{H})$ stretching modes, symmetric and degenerate, respectively [19]. Our data are in accordance with those reported in literature.

The peak around $3331 \mathrm{~cm}^{-1}$ is determined by the existence of water-bonded molecules in the TEA solution resulting from the synthesis process. The vibrations of these molecules are modified by the bonds with TEA, fact that determines the shift of the main peak of pure water from $3453 \mathrm{~cm}^{-1}$ towards $3331 \mathrm{~cm}^{-1}$. The presence of water is confirmed also by the existence of the peak at $1642 \mathrm{~cm}^{-1}$. To support this idea we added supplementary water in proportion of $10 \%$ to pure TEA and we recorded again the spectrum. We can observe modification of the shape of the band around $3331 \mathrm{~cm}^{-1}$ compared with the similar bands of pure water and pure TEA. The band $1642 \mathrm{~cm}^{-1}$ does not shift but its intensity increases in sample with water added in excess (Fig. 3).

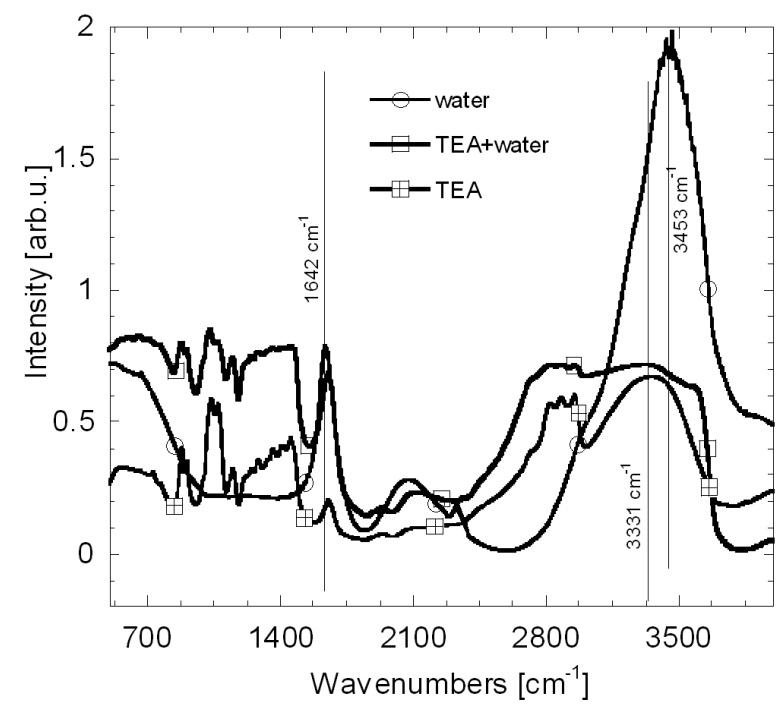

Fig. 3. IR spectra of pure TEA, pure water, and TEA with surplus of water.

Detailed explications of the effect of different environments on the $\mathrm{OH}$ stretching vibration of water, based on the Raman analysis of the large band around $3453 \mathrm{~cm}^{-1}$, are given in many references. In agarose environment, this band was fitted with five Gaussian peaks, around $3050,3200,3400,3500$, and $3600 \mathrm{~cm}^{-1}$ [20]. In different kind of pure and saline water this band was decomposed in five-Gaussian components centered at 3018, $3223,3393,3506$, and $3624 \mathrm{~cm}^{-1}$. From these studies results that the three components on the low frequency side are assigned to hydrogen-bonded water, while the remaining two high frequency side components are assigned to non-hydrogen-bonded water [21]. 


\subsubsection{Raman analysis of pure $P A A$}

Other information concerning the molecular vibration is obtained by the Raman spectroscopy $[22,23]$. The Raman spectrum of pure PAA, in solid state, shows some intense and large bands located in the domain 400$1800 \mathrm{~cm}^{-1}$, associated with the different possible local conformations of the polymeric chain, determined by the hydrogen bonds (Fig. 4). The hydrogen bonds appear between the $(-\mathrm{OH})$ group and the oxygen atom of two different carboxylic groups belonging to two different chains or to the same chain. Many arrangements are possible, linear structure with the carboxyl groups included in cyclic dimers (the dimer is formed by hydrogen bonds between two carboxylic groups of adjacent molecules); laterally hydrogen-bonded structure (when the $\mathrm{COOH}$ groups involved in the hydrogen bond belong to the same chain but are situated on the same side of the chain); the face-on bonded structure (where the carboxylic groups are face to face but each group of one chain is connected with two other different similar groups of other neighboring chain) [12]. The assignment of the Raman bands are listed in Table [15]. As we mentioned previously,

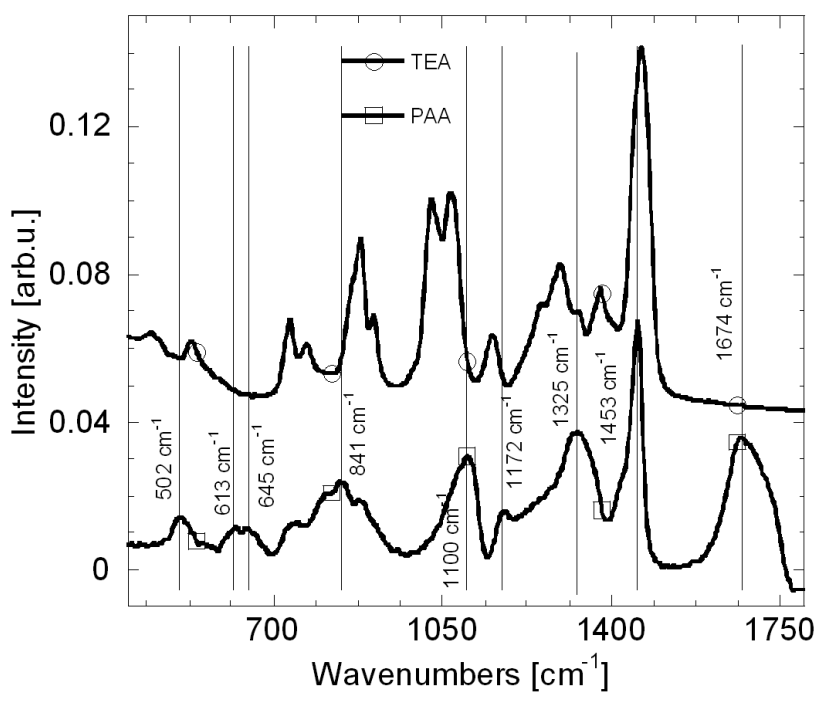

Fig. 4. Raman spectra of PAA and TEA in pure state.

some bands, which are IR active, can be seen also in the Raman spectrum. For instance the band $629 \mathrm{~cm}^{-1}$ is included in the large Raman band centered at $645 \mathrm{~cm}^{-1}$; the IR band at $1172 \mathrm{~cm}^{-1}$ appears as shoulder in the Raman spectrum at the same wave number; the IR band at $1446 \mathrm{~cm}^{-1}$ can be seen at $1453 \mathrm{~cm}^{-1}$ in the Raman spectrum.

\subsubsection{Raman analysis of pure TEA}

The Raman spectrum of pure TEA contains vibration bands as follows: a sharp and intense band at $1461 \mathrm{~cm}^{-1}$. This band is close to the band at $1452 \mathrm{~cm}^{-1}$ of pure PAA. A sharp small peak at $1375 \mathrm{~cm}^{-1}$, three bands close each to other at 1325,1290 , and $1252 \mathrm{~cm}^{-1}$ with the highest peak at $1290 \mathrm{~cm}^{-1}$. The band at $1325 \mathrm{~cm}^{-1}$ appears also
TABLE

Assigment of the Raman bands.

\begin{tabular}{c|c|c}
\hline $\begin{array}{c}\text { Wave numbers } \\
{\left[\mathrm{cm}^{-1}\right]}\end{array}$ & Description & Assignment \\
\hline 1674 & large & $\nu(\mathrm{C}=\mathrm{O})$ dimer \\
1453 & narrow & $\delta\left(\mathrm{CH}_{2}\right)$ \\
1325 & large & $\nu_{\text {as }}(\mathrm{CCO})$ \\
1100 & large & $\rho\left(\mathrm{CH}_{2}\right)$ \\
841 & large, with many & $\nu_{\mathrm{s}}(\mathrm{CCO}), \tau\left(\mathrm{CH}_{2}\right)$ \\
645 & shoulders & $\delta(\mathrm{C}=\mathrm{O})$ cis \\
613 & shoulder & $\delta(\mathrm{C}=\mathrm{O})$ trans \\
502 & shoulder & $\delta(\mathrm{CCO})$ cis
\end{tabular}

in the spectrum of pure PAA. Clearly separated peaks appear at 1152, 1064 and $1024 \mathrm{~cm}^{-1}$; two peaks are very close each to other at 907 and $877 \mathrm{~cm}^{-1}$; two small peaks at 767 and $730 \mathrm{~cm}^{-1}$, small peaks at 526 and $443 \mathrm{~cm}^{-1}$ (Fig. 4). Assignment of these bands can be done on the basis of results obtained by Koruda et al. on similar systems [19]. In the following sections our attention was focused on the band at $1290 \mathrm{~cm}^{-1}$ attributed to (CCO), and of the band at $794 \mathrm{~cm}^{-1}$ attributed to $\left(\mathrm{CH}_{2}\right)$ groups, which are the most affected by the presence of water and neutralizer [12, 19].

\subsection{Analysis of the aqueous gels}

\subsubsection{IR analysis}

The polymeric gels are characterized by the existence of two separate phases, the polymer network and the solvent. In aqueous PAA gels the main effect of the solvent is the perturbation of the hydrogen bonds established between the carboxylic groups [12]. The effect depends on the concentration of water. At low water concentration the interchain hydrogen bonds are strong enough to avoid their breakage by the water molecules. The water is trapped only in the limited free volume of the polymer network (Fig. 1c). When the concentration increases the hydrogen bonds can be partially destroyed and the water molecules can be included into cyclic dimers (Fig. 1d), or into three-dimensional structures (open dimers or oligomers) [12]. Other possibility is the attachment of water molecules to terminal chain carboxylic groups not included in hydrogen bonds (Fig. 1e). The formation of these species leads to the modification of vibration in the range of $1640-1714 \mathrm{~cm}^{-1}$. The last two situations correspond to our samples, because they have great content of water. The most important change after hydration concerns the band attributed to cyclic dimers, which appears at $1714 \mathrm{~cm}^{-1}$ in the spectrum of pure PAA in powder state. In the gels this band appears as a shoulder at $1714 \mathrm{~cm}^{-1}$ of a large band with the peak at $1642 \mathrm{~cm}^{-1}$. The shoulder is more evident for the gel with $1.5 \%$ PAA concentration and less pronounced at $1 \%$ and $0.5 \%$ concentration (Fig. 5 ). This behavior can be 
correlated with the decrease of the polymeric concentration in these gels.

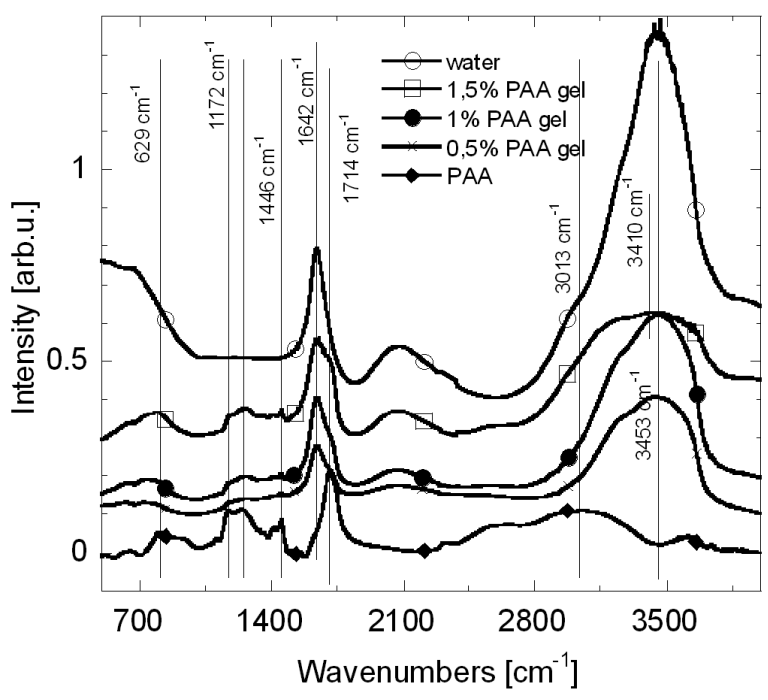

Fig. 5. IR spectra of pure water, pure PAA and of the non-neutralized PAA gels with the concentrations 0.5 , 1 , and $1.5 \%$.

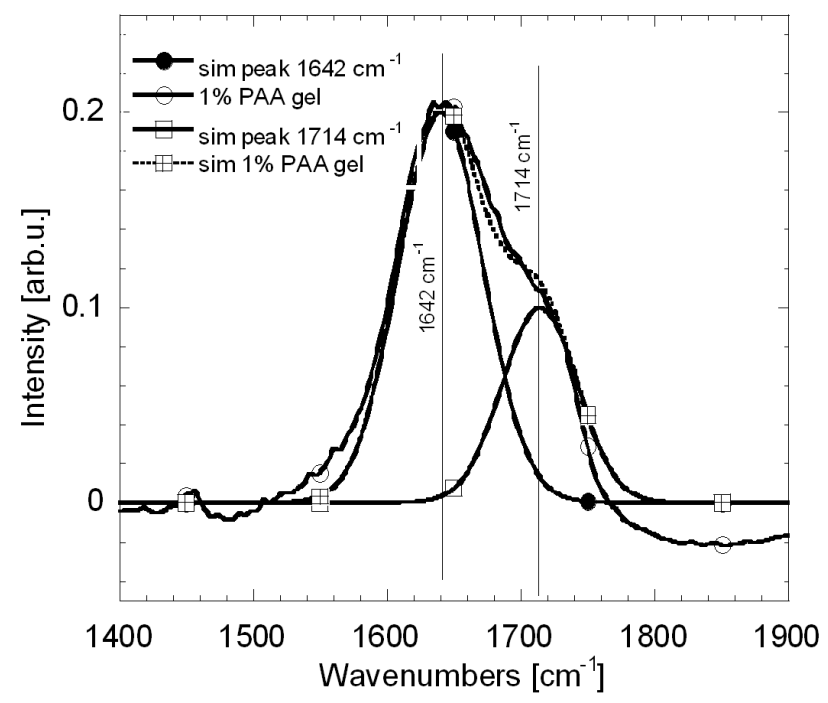

Fig. 6. Deconvolution of the peak $1642-1714 \mathrm{~cm}^{-1}$ of the $1 \%$ PAA gel.

The variation of water concentration on these gels is very small, from $99.5 \%$ to $98.5 \%$, whereas the concentration of polymer decreases 3 times for the $1.5 \%$ gel to $0.5 \%$. The pure water gives rise to the band at $1642 \mathrm{~cm}^{-1}$ and the pure PAA the band at $1714 \mathrm{~cm}^{-1}$. The large band $1640-1714 \mathrm{~cm}^{-1}$, being a superposition of water and PAA bands, its shape and amplitude depend on the contribution of both components. The contribution of each component can be established only after the deconvolution of the spectra. Such example for the gel $1 \%$ is shown in Fig. 6.

In the gels some of the carboxyl groups interact with water forming open dimers or oligomers [12]. As the concentration of polymer decreases the evolution towards open dimers is favorite that explains the shift towards $1642 \mathrm{~cm}^{-1}$ [24].

Other visible effect is the apparition of large band around $3450 \mathrm{~cm}^{-1}$. This band does not appear in the spectrum of dried polymer. In the spectra of the gels this band shifts slowly from $3453 \mathrm{~cm}^{-1}$ at $0.5 \%$ PAA concentration, to $3410 \mathrm{~cm}^{-1}$ at $1.5 \%$ (Fig. 5). Studies performed on similar materials suggest that this band is caused by symmetric stretching vibration of hydrogen bonded water molecules [25]. These vibrations are sensitive to the water content of the gels, idea supported by the monotonical shift of this band with the concentration of the gels. The bands at $1446 \mathrm{~cm}^{-1}$, assigned to $\delta\left(\mathrm{CH}_{2}\right), 1241,1172$, and $629 \mathrm{~cm}^{-1}$ assigned to $\delta(\mathrm{C}=\mathrm{O}$, trans) [15], which appear in the spectrum of dried polymer, appear at the same wave numbers in the gels, with small amplitude. However, their intensity increases monotonically with the concentration of polymer (Fig. 5). The band at $1446 \mathrm{~cm}^{-1}$ has low intensity in the gels due to the increased flexibility of the polymer backbone after the destruction of hydrogen bonds [12]. The decrease of the intensity of the bands 1241 and $1172 \mathrm{~cm}^{-1}$ is determined by the decay in the number of hydrogen bonds in gels, and possible coupling of the $(\mathrm{C}=\mathrm{O})$ stretching and $(-\mathrm{OH})$ bending vibrations [12]. The band at $2350 \mathrm{~cm}^{-1}$ appears at the same wave number in the polymer and in the gels. The difference between the spectra of the powder polymer and the gel (i.e. the shift of the band $1714 \mathrm{~cm}^{-1}$ of pure polymer towards $1642 \mathrm{~cm}^{-1}$ for the gel), the apparition of the band $3450 \mathrm{~cm}^{-1}$ in the spectrum of the gel, the dependence of the intensity of these bands on the concentration of the polymer show the modifications of the vibration modes after hydration. These modifications are determined by the perturbation of hydrogen bonds.

\subsubsection{Raman analysis}

The perturbation of the hydrogen bonds of PAA by the water molecules in the gels is supported also by the Raman investigation. The Raman spectra of the gels show some differences with respect to pure PAA, some bands almost vanish and some shift towards low wave numbers (Fig. 7). For instance the band $1674 \mathrm{~cm}^{-1}$ shifts to $1642 \mathrm{~cm}^{-1}$. This shift is determined by the destruction of hydrogen bonds and incorporation of water molecules into cyclic dimers [12]. The band at $1453 \mathrm{~cm}^{-1}$ assigned to $\delta\left(\mathrm{CH}_{2}\right)$ and the band at $1325 \mathrm{~cm}^{-1}$ assigned to $\nu_{\text {as }}(\mathrm{CCO})$ of pure PAA can be hardly seen in the spectrum of the gel. The band $841 \mathrm{~cm}^{-1}$, associated to $\tau\left(\mathrm{CH}_{2}\right)$, shifts to $797 \mathrm{~cm}^{-1}$.

At high concentration of the water (as in our samples), many possibilities of inclusion of water into the polymeric network appear. The water can be included into the cyclic dimers formed by $(-\mathrm{COOH})$ groups. Dipolar interactions appear between the water molecules and the ($\mathrm{OH})$ parts of carboxylic groups. Due to the great content of water in these gels we can suppose that the majority of these bending groups are included in new bonds with water molecules. In the gels very few bending $(-\mathrm{OH})$ groups 
remain in the same environment as in the pure polymer, fact that can be correlated with the decrease in intensity

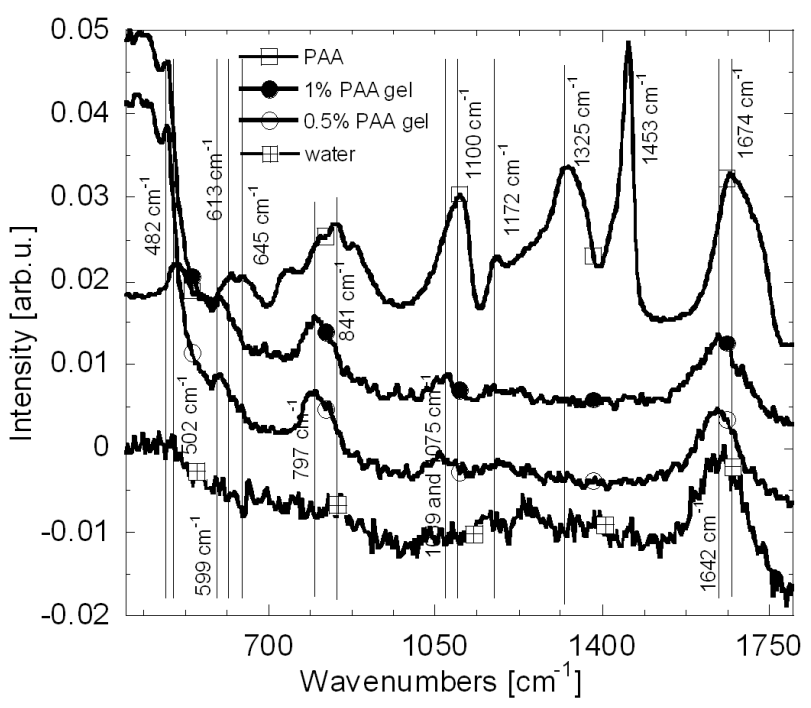

Fig. 7. Raman spectra of pure water, solid PAA and aqueous PAA gels with the concentrations 0.5 and $1 \%$.

of the bands $1325 \mathrm{~cm}^{-1}$ and $1453 \mathrm{~cm}^{-1}$. Other possibility for water molecules is to occupy positions outside the carboxylic groups, in the vicinity of $\left(\mathrm{CH}_{2}\right)$ groups, or in the free spaces determined by the arrangement of the polymeric chains. This situation can be correlated with the shift of the band $841 \mathrm{~cm}^{-1}$ to $797 \mathrm{~cm}^{-1}$ [12]. The band $1110 \mathrm{~cm}^{-1}$, assigned to $\rho\left(\mathrm{CH}_{2}\right) \mathrm{cis}$, shifts to $1059-1075 \mathrm{~cm}^{-1}$, and the large band $613-645 \mathrm{~cm}^{-1}$, assigned to $\delta(\mathrm{C}=\mathrm{O})$ cis, shifts to $599 \mathrm{~cm}^{-1}$. The band $502 \mathrm{~cm}^{-1}$ shifts to $482 \mathrm{~cm}^{-1}$. These modifications were observed for all the gels, without notable differences between the samples with different concentrations. The difference of polymeric concentration between these samples is too small in order to observe a significant effect of the variation of the polymeric concentration. As we can see from Fig. 5 the difference between the spectra of samples 0.5 and $1 \%$ is only a small variation of the intensity of the bands.

Due to the small quantity of PAA and due to the noise all the vibration bands of the PAA can be hardly seen on the spectra of the gels.

Comparison of the Raman spectra of pure PAA, pure water and PAA gels confirms the influence of water on the polymeric conformation (Fig. 7).

\subsection{Neutralized samples}

\subsubsection{IR analysis}

The neutralization of the PAA is necessary for two reasons, annihilation of the acid character of the polymer and increase of the viscosity. Neutralization can be easy done with common bases as $\mathrm{NaOH}$ or TEA. Optimum neutralization is achieved at $\mathrm{pH}$ of $6.5-7.0$, but is not necessary for all medical applications to reach this value. Neutralization with TEA is followed by the increase of the viscosity. Generally the neutralization is conducted until a desired viscosity is obtained, even the $\mathrm{pH}$ does not achieve the optimum value [7]. As demonstrated by other authors the high viscosities can be obtained in a $\mathrm{pH}$ range of 5.0-9.0. This corresponds to neutralization ratio $1.5 / 1$ base/polymer [26]. The ammonia group of TEA interacts with the acid sites of the polymer destroying the interchain hydrogen bonds. In this reaction the $\mathrm{H}$ atom of $(-\mathrm{COOH})$ group is included in $\left(\mathrm{NH}_{4}\right)$ group (Fig. 1b). The vibrations of the carboxylic groups are affected by these new bonds.

As consequence the band $1714 \mathrm{~cm}^{-1}$ of pure PAA shifts at $1642 \mathrm{~cm}^{-1}$ for the neutralized gels (Fig. 8). If in the non-neutralized gels we can observe the band at $1714 \mathrm{~cm}^{-1}$ as a shoulder of the IR spectrum, in the neutralized samples this band is completely shifted at $1642 \mathrm{~cm}^{-1}$ (Fig. 8). The band $1642 \mathrm{~cm}^{-1}$ can be observed in the spectrum of pure TEA (Fig. 2). This shift suggests the idea that almost all the carboxylic groups were neutralized by the base [12]. In this figure the spectra are presented for pure PAA and TEA as well as the spectra of neutralized gel $1 \%$ and the same gel neutralized. Other bands of pure PAA can be hardly seen in both spectra, neutralized and non-neutralized due to the absorption of water molecules. The bands of pure TEA appear in the spectrum of neutralized samples without shift, but their amplitudes depend on the concentration of polymer. For instance the bands 879 and $905 \mathrm{~cm}^{-1}$ are almost missing at $0.5 \%$ and $1 \%$ concentrations, but they appear at $1.5 \%$.

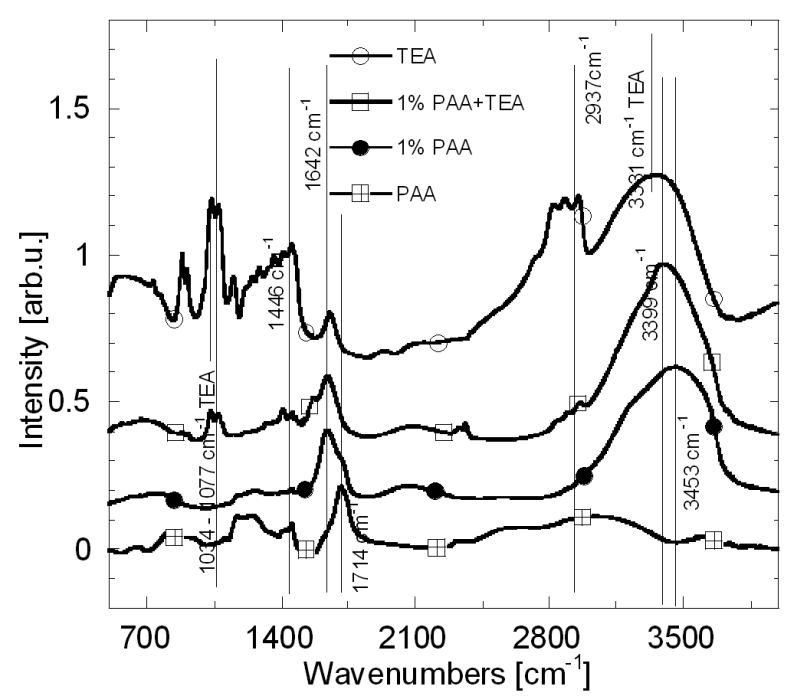

Fig. 8. Comparison between the IR spectra of pure PAA, pure TEA, 1\% PAA aqueous gel and 1\% PAA neutralized gel.

The same situation concerns the bands in the domain 1034-1077 $\mathrm{cm}^{-1}$, and the bands of the domain 2810 and $2965 \mathrm{~cm}^{-1}$ (Fig. 9). The content of TEA is greater for the gels with great concentration of PAA because the ratio $\mathrm{PAA} / \mathrm{TEA}$ is the same for all the samples. The concentration of TEA increases when the concentration of polymer increases. This fact explains the dependence of 
the amplitude of TEA's bands with the concentration of the gels. Other important modification concerns the large band around $3400 \mathrm{~cm}^{-1}$. The pure TEA shows a peak at $3331 \mathrm{~cm}^{-1}$ determined by the bonded water. For neutralized samples the maximum of this band is observed at $3332 \mathrm{~cm}^{-1}$ for $1.5 \%$, at $3399 \mathrm{~cm}^{-1}$ for $1 \%$ and at $3442 \mathrm{~cm}^{-1}$ for $0.5 \%$ of PAA (Fig. 9). This shift follows the variation of the concentration of TEA. At high TEA concentration (sample with concentration 1.5\%), this band is close to those of pure TEA, and shifts towards high wave numbers when the concentration of TEA decreases (samples with concentration $0.5 \%$ ). The band around $3400 \mathrm{~cm}^{-1}$ cannot be observed in the spectrum of pure PAA but it appears in the spectra of the gels. For the neutralized gels this band is determined by the overlap of the band $3453 \mathrm{~cm}^{-1}$ of pure water and the band $3331 \mathrm{~cm}^{-1}$ of pure TEA. The shift of the maximum of this band is determined by the variation of the concentration of bonded-water of TEA with the concentration of the gels.

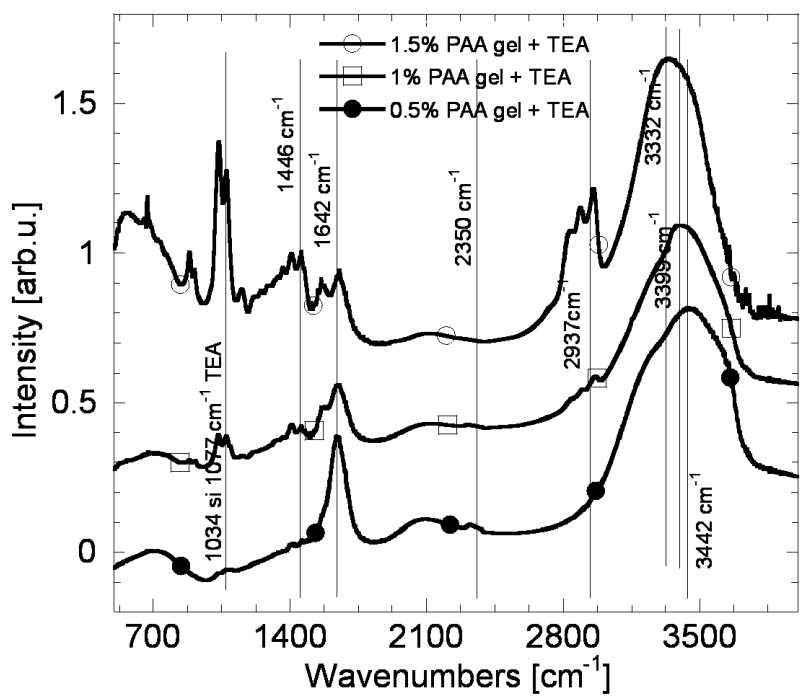

Fig. 9. IR spectra of neutralized gels with the polymeric concentrations $0.5,1$, and $1.5 \%$.

\subsubsection{Raman analysis}

The Raman spectra of the neutralized samples show some similitude with the spectra of non-neutralized gels but also differences, determined especially by the presence of the TEA. For instance, the band $1642 \mathrm{~cm}^{-1}$ of the gels without TEA appears in both the spectra without modification (Fig. 10). The bands between 1059 and $1075 \mathrm{~cm}^{-1}$ of the gels without TEA are superposed on the bands $1024 \mathrm{~cm}^{-1}$ and $1064 \mathrm{~cm}^{-1}$ of pure TEA. Other bands appearing in the spectra of neutralized gels belong to TEA. The band $1461 \mathrm{~cm}^{-1}$ of pure TEA appears without shift in the spectra of the gel. The large band with the highest peak at $1290 \mathrm{~cm}^{-1}$ of pure TEA shifts to $1317 \mathrm{~cm}^{-1}$ in the spectrum of the neutralized gel and becomes large. The bands 1064, 1024, 907 and $877 \mathrm{~cm}^{-1}$ of pure TEA appear without shift in the neutralized gels, but their amplitudes are reduced because the concentration of TEA in the gels is small compared with the pure TEA. The bands 730 and $767 \mathrm{~cm}^{-1}$ of pure TEA merge into a large band with a peak at $740 \mathrm{~cm}^{-1}$ in the spectrum of the neutralized gel. This band is superposed on the band $794 \mathrm{~cm}^{-1}$ of non-neutralized gel. Practically only the bands at $1290 \mathrm{~cm}^{-1}$, assigned to $\nu_{\text {as }}(\mathrm{CCO})$, and $794 \mathrm{~cm}^{-1}$, assigned to $\tau\left(\mathrm{CH}_{2}\right)$, are affected by neutralization. That means modifications of the vibrations of the $(\mathrm{CCO})$ and $\left(\mathrm{CH}_{2}\right)$ groups, determined by local rearrangement of the polymeric conformation after neutralization. However some amount of polymer and neutralizer remains in initial state, without chemical reaction between them, fact that explains the existence of vibration bands of pure TEA without modification in the spectrum of neutralized gels.

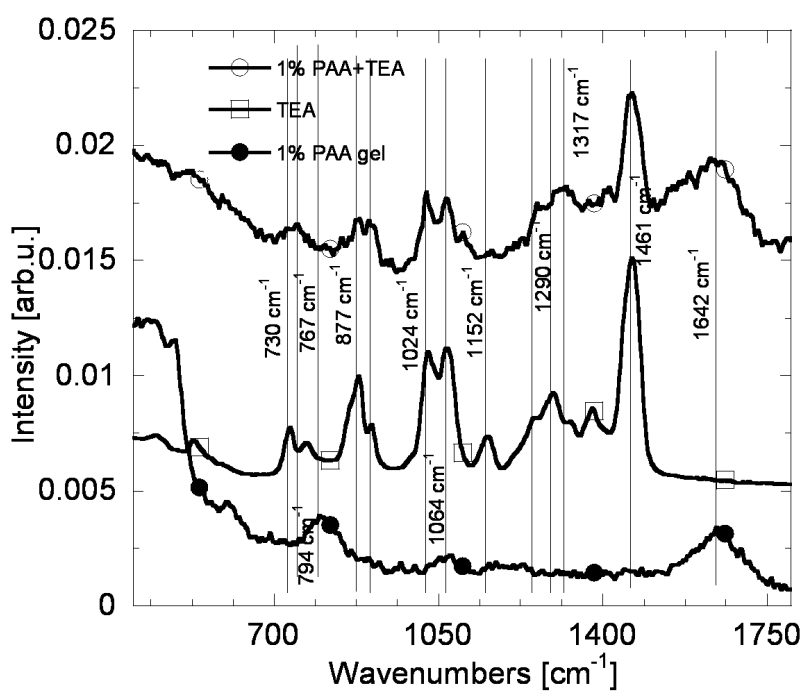

Fig. 10. Comparison between the Raman spectra of pure TEA, 1\% PAA aqueous gel, and 1\% PAA neutralized gel.

\section{Conclusion}

The modifications induced on molecular scale by the hydration of PAA, and neutralization with TEA of the PAA gels, were observed by IR and Raman spectroscopy. In solid state the PAA is characterized by coiled conformation of the chains, with low degree of interconnections between them. Hydration of PAA has as effect the perturbation of the hydrogen bonds established between the carboxylic groups of the polymer, and the evolution of the local conformation of the polymer towards uncoiled conformation. At high concentration of water the hydrogen bonds are partially destroyed and the water molecules are included into cyclic dimers. This effect is associated with the shift of the band of the carbonyl group of the carboxylic group from $1714 \mathrm{~cm}^{-1}$ to $1642 \mathrm{~cm}^{-1}$ in the IR spectra. In the Raman spectra this effect is associated with the shift of the band $1674 \mathrm{~cm}^{-1}$ of solid PAA to $1642 \mathrm{~cm}^{-1}$ for the gels. Other visible effect is the apparition in the IR spectra of the gels of the large band 
in the domain $3500-3400 \mathrm{~cm}^{-1}$ which change its shape when the concentration of the polymer increases. This band is associated with the existence of water clusters in the vicinity of the polymeric chains. Other possibility is the inclusion of water molecules in the free spaces determined by the arrangement of the polymeric chains or the positioning near the $\left(\mathrm{CH}_{2}\right)$ groups. This situation is correlated to the shift of the Raman band $841 \mathrm{~cm}^{-1}$ of pure PAA to $797 \mathrm{~cm}^{-1}$ for the gels. On the macroscopic scale the hydrations of PAA change dramatically the mechanical properties of the polymer, which passes from fragile state, the powder, into a more flexible state, the gel.

The neutralization with TEA affects the acid sites of the polymer and destroys a part of the hydrogen bonds. The consequence is the shift of the IR band from $1714 \mathrm{~cm}^{-1}$ to $1642 \mathrm{~cm}^{-1}$. Other effect of the neutralization is the modification of the large IR band 3400$3500 \mathrm{~cm}^{-1}$ of the gels after neutralization. This behavior is determined by the overlap of the bands of bondedwater molecules of TEA and the band or pure water.

The perturbation of carboxylic groups which are involved in chemical reaction with TEA, is confirmed by the shift of the Raman bands located around $1290 \mathrm{~cm}^{-1}$ and $794 \mathrm{~cm}^{-1}$.

The major effect of hydration and neutralization is the perturbation of the hydrogen bonds established between the neighboring polymeric chains followed by the modification of the local polymeric conformation.

\section{References}

[1] A.S. Hoffman, Adv. Drug Del. Rev. 43, 3 (2002).

[2] P. Sriamornsak, R.A. Kennedy, Int. J. Pharm. 323, 72 (2006).

[3] J.S. Ahn, H.K. Choi, M.K. Chun, J.M. Ryu, J.H. Jung, Y.U. Kim, C.S. Cho, Biomaterials 23, 1411 (2002).

[4] M.K. Chun, C.S. Cho, H.K. Choi, J. Appl. Polym. Sci. 79, 1525 (2001).

[5] M.K. Chun, C.S. Cho, H.K. Choi, J. Control. Rel. 81, 327 (2002).

[6] S. Rossi, G. Sandri, F. Ferrari, M.C. Bonferoni, C. Caramella, Pharm. Dev. Technol. 8, 199 (2003).

[7] C. Tiu, J. Guo, P.H.T. Uhlherr, J. Ind. Eng. Chem. 12, 653 (2006).
[8] E. Dinte, S.E. Leucuta, Farmacia LII 5, 13 (2004).

[9] J.L. Koenig, A.C. Angood, J. Semen, J.B. Lando, J. Am. Chern. Sot. 91, 26, 7250 (1996).

[10] J. Dong, Y. Ozaki, K. Nakashima, Macromolecules 30, 1111 (1997).

[11] B. Stuart, Polymer Analysis, Wiley, Chichester 2002.

[12] M. Hoerter, A. Oprea, N. Bârsan, U. Weimar, Sens. Actuat. B 134, 743 (2008).

[13] J.P. Chen Addad, Physical Properties of Polymeric Gels, Wiley, Chichester 1996.

[14] Tae Hee Kim, Jae Soon Ahn, Hoo Kyun Choi1, Yun Jaie Choi, Chong Su Cho, Arch. Pharm. Res. 30, 381 (2007).

[15] K. Itoh, M. Yaita, T. Hasegawa, S. Fujii, Y. Misono, J. Electron Spectrosc. Relat. Phenom. 54-55, 923 (1990).

[16] He Chen, Jiaxing Li, Dadong Shao, Xuemei Ren, Xiangke Wang, Chem. Eng. J. 210, 475 (2012).

[17] K. Ichikawa, T. Mori, H. Kitano, M. Fukuda, A. Mochizuki, M. Tanaka, J.'Polym. Sci. B Polym. Phys. 39, 2175 (2001).

[18] N. Tanaka, H. Kitano, N. Ise, J. Phys. Chem. 94, 6290 (1990).

[19] Y. Koruda, M. Kimura, Spectrochim. Acta 22, 47 (1966).

[20] B. Ratajska-Gadomska, W. Gadomski, J. Chem. Phys. 121, 12583 (2004).

[21] Hsiao-Chien Chen, Hsiu-Chen Lin, Hsi-Hsien Chen, Fu-Der Mai, Yu-Chuan Liu, Chun-Mao Lin, ChunChao Chang, Hui-Yen Tsai, Chih-Ping Yang, Sci. Rep. 4, 4425 (2014).

[22] R.J. Young, P.A. Lovell, Introduction to Polymers, Chapman \& Hall, London 1991.

[23] M. Baia, S. Astilean, T. Iliescu, Raman and SERS Investigations of Pharmaceuticals, Springer-Verlag, Berlin 2008.

[24] M.T. Islam, N. Rodriguez-Hornedo, S. Ciotti, Ch. Ackermann, The AAPS J. 6, 61 (2004).

[25] K. Ichikawa, T. Mori, H. Kitano, M. Fukuda, A. Mochizuki, M. Tanaka, J. Polym. Sci. B Polym. Phys. 39, 2175 (2001).

[26] Noveon Bulletin, TDS-237, Edition: January 2002. 